which would explain these data as well as the Spector and Hull findings, is that the olfactory bulb is the locus-or at least one essential locus-of the inhibitory command. That is, an inhibitory mechanism must involve some anatomical loci and-without any direct evidence in either direction-it seems not unreasonable to assume that some aspect of the inhibition system may be damaged by olfactory bulb removal. Such removal would therefore render the inhibition system inoperative, such that odors, even if they could be processed, would be irrelevant. This formulation can thus account for all the otherwise puzzling phenomena in this area, and furthermore suggests an evolutionary mechanism for the acquisition of the inhibitory function.

\section{REFERENCES}

Bugbee, N. M., \& Eichelman, B. S., Jr. Sensory alterations and aggressive behavior in rat. Physiology \& Behavior, 1972, 8, 981-985.

Karli, P. Vergnes, M \& Didiergeorges, F Rat-mouse interspecific aggressive behaviour and its manipulation by brain ablation and $\mathrm{by}$ brain stimulation. In $\mathrm{S}$. Garrattini and E. B. Sigg (Eds.), Aggressive behavior, proceedings of the international symposium on the biology of aggressive behavior. held at the Instituto di Richerche Farmacologiche Mario Negri, Milan, 1968. New York: Wiley, 1969.

Malick, J. B. A b ehavioral comparison of three lessions-induced models of aggression in the rat. Physiology \& Behavior, 1970 , 5, 679-681.

Myer, J. S. Stimulus control of mouse-killing rats. Journal of Comparative \& Physiological Psychology, 1964, 58, 112-117. Siegel, S. Nonparametric statistics for the behavioral sciences. New York: McGraw-Hill, 1956.

Spector, S. A., \& Hull, E. M. Anosmia and mouse killing by rats: A nonolfactory role for the olfactory bulbs. Journal of Comparative \& Physiological Psychology, 1972, 80, 354-356. (R eceived for publication July 17, 1974.)

\title{
Ontogenetic changes in classification behavior
}

\author{
HOWARD H. KENDLER and JOAN HELLAND \\ University of California, Santa Barbara, California 93106
}

\begin{abstract}
A developmental study of free-classification behavior within the age range of $3-1 / 2$ to 19 years indicates that categorical responses, which are characteristic of adult behavior, increase with age while overgeneralized responses, classifications including noncategorical instances, decrease with age. Overdiscriminated responses which are incomplete categorical classifications increase from $3-1 / 2$ to 6 years and then decrease to 19 years of age. These results are discussed within a two-stage theory of conceptual development (Kendler, 1971).
\end{abstract}

This paper reports selected findings from a large-scale developmental study that was designed to investigate the two-stage theory of conceptual development (Kendler, 1971). This formulation, which is an outgrowth of discrimination shift studies (Kendler \& Kendler, 1962; 1970), postulates that, at early ages, the mechanism of stimulus generalization guides classsification performance while later on mediational mechanisms in the form of implicit representational responses control such performance. During the early period of the mediational stage, it is additionally assumed that Ss form narrow concepts and only later do they become capable of forming broad concepts that are characteristic of adult behavior (Saltz \& Siegel, 1967).

\section{METHOD}

\section{Subjects}

A total of 192 Ss, 32 at six different age levels, are described in Table 1. An additional 22 Ss were eliminated, 3 for E's error; 16 Ss from the three youngest age levels who refused to finish the experiment, 2 who failed to complete the experiment due to illness, and one 3-year-old who could not solve all the practice problems. The 3- and 4-year-old children were drawn from private nursery schools; the elementary school child ren, from the public school system in Goleta, California, and the college students from introductory psychology classes at the University of California, Santa Barbara. Analysis of variance of the children's Peabody Picture Vocabulary Test (PPVT) scores (Dunn, 1959) failed to indicate any significant age-related difference in intelligence over age $[F(4,155)=1.47, \mathrm{p}>.20]$.

\section{Procedure}

Each S was confronted with a sequence of eight successive classification tasks, four of which involved two-dimensional stimuli (Fig. 1), the results of which are reported in this paper. The remaining four tasks involved stimulus patterns with binary values of four characteristics (e.g., a schematic drawing of a human face with two values of the eyes, nose, mouth, and moustache). The results obtained with these stimuli will not be reported because of unanticipated and insuperable difficulties in developing satisfactory response measures.

Table 1

Description of Ss at Each Age Level

\begin{tabular}{rrrrrrrr}
\hline \multicolumn{2}{c}{ Age in Months } & & \multicolumn{2}{c}{ Number } & & \multicolumn{2}{c}{ PPVT IQ } \\
\cline { 6 - 7 } Mean & Range & & Male Female & & Mean & SD \\
\hline 42.4 & $36-47$ & & 14 & 18 & & 115.9 & 13.5 \\
54.1 & $49-59$ & & 14 & 18 & & 112.6 & 9.9 \\
69.5 & $65-76$ & & 12 & 20 & & 112.2 & 7.2 \\
97.4 & $85-110$ & 18 & 14 & & 109.3 & 13.5 \\
144.2 & $131-156$ & & 15 & 17 & & 110.0 & 14.8 \\
225.7 & $215-247$ & 9 & 23 & & - & - \\
\hline
\end{tabular}


Each $\mathrm{S}$ was tested individually in a quiet room. The children were given the Peabody Picture Vocabulary Test. The experiment proper began with a sequence of three practice problems. Three boxes, two black and one gray, with an opening in the top through which response cards could be inserted were placed in front of the S. A stimulus card was displayed on the front of each black box. For the first practice problem one black box had a small red circle, the other a large red square. Eleven response cards (four squares of two different sizes and four different colors, four circles of two different sizes and four different colors, two triangles of different sizes and colors, and a multicolored irregular shape) were displayed in a random pattern in front of the $\mathrm{S}$. The $\mathrm{S}$ was instructed to sort the cards into one of the three boxes; the stimulus card on each black box indicating which response cards should be inserted in it. Cards that did "not belong" or "were not like" the stimulus cards of either black box were to be deposited into the gray box. The $\mathrm{E}$ emphasized that a response card did not have to be identical to a stimulus card in order to be correctly placed in that box. The $S$ was instructed to pick up one response card at a time and deposit it in the box of his choice. Ss were trained by positive and negative feedback to sort all the squares in the black box that displayed the stimulus card with the large red square, all circles in the other black box with the small red circle, and the two triangles and the irregular shape in the gray box. Each $\mathrm{S}$ was given five trials with the entire set of 11 response cards to achieve a correct sort without any errors. Two similar practice problems followed and then the main task began.

The experimental tasks were conducted under a free classification procedure in which all the response cards were displayed randomly in front of $S$ who sorted them in a self-paced manner into what he considered to be the appropriate boxes. Half of the experimental tasks required the $S$ to discriminate between three sorting categories represented by the two black boxes with different stimulus cards plus the gray box. In half of these tasks the two stimulus cards differed in only one dimension (e.g., the two narrowly separate angles, one of which is pointed and the other rounded in Fig. 1), while in the remaining half the two stimulus cards differed in both dimensions (e.g., a narrowly separated pointed angle and a widely separated rounded angle). The remaining half of the experimental tasks required $S$ to discriminate between only two categories; one black box with a stimulus card and the gray box.

Orthogonal to the variable of number of sorting categories were two memory conditions; presence or absence of the stimulus card(s) during sorting. For half of the problems, the stimulus cards were present throughout the sorting task while for the remaining ones the stimulus cards were removed immediately prior to sorting. In the latter case, the $\mathrm{S}$ was permitted to look at stimulus cards as long as he desired before presentation of the response cards.

The two youngest age groups required two experimental sessions, usually on successive days to complete all the sorting problems, while the other Ss finished their one task in one session.

\section{RESULTS}

The sorting behavior of each $\mathrm{S}$ for each problem was characterized in one of four ways: categorical (C), overdiscrimination (OD), overgeneralization (OG), and unclassifiable (UC). A categorical response, using the pointed and rounded angles in Fig. 1 as illustrations, would be achieved if the $S$ in the two stimulus card condition classified all the pointed angles together in the appropriate black box and the rounded angles in the other. In the case where the two stimulus cards differed in two characteristics (pointedness and width) then a
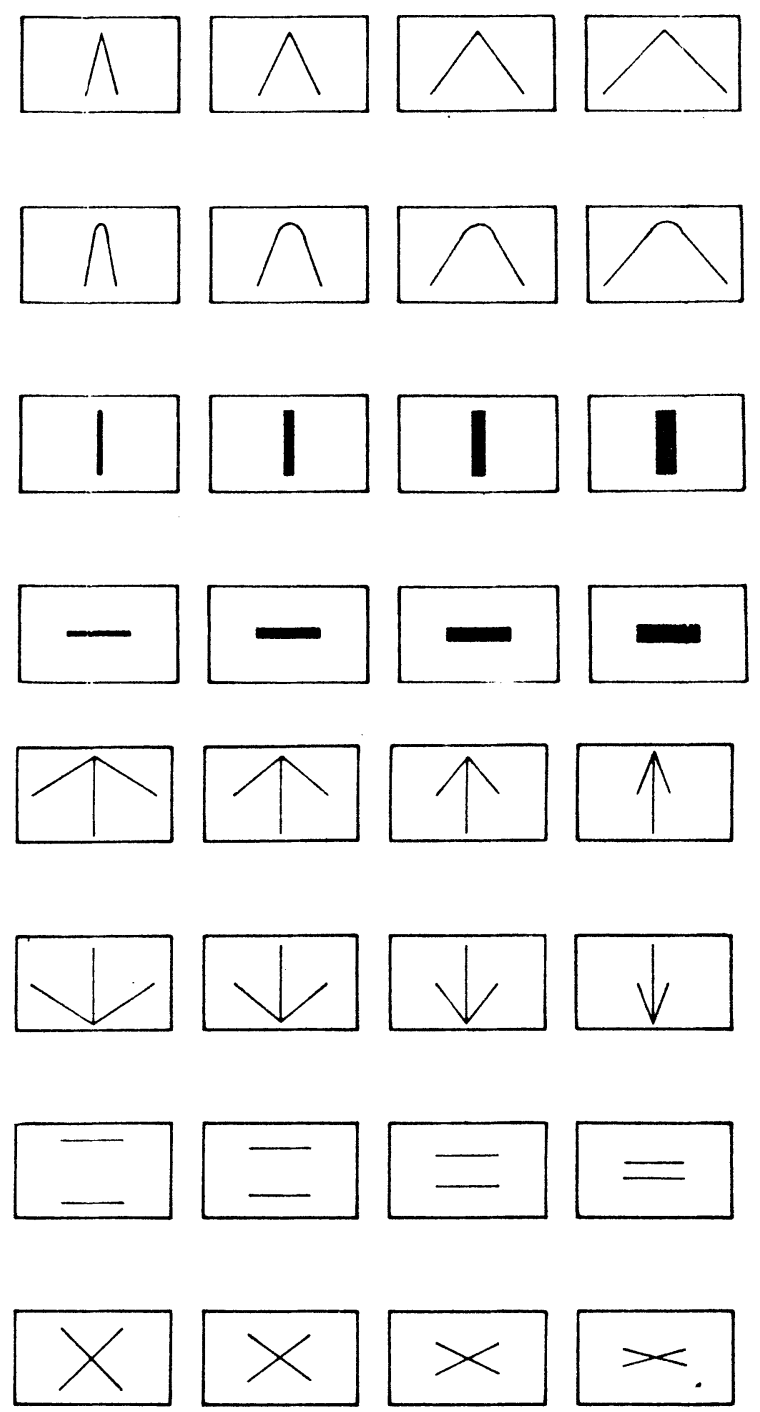

Fig. 1. The two-dimensional stimuli used in the classification tests. Each successive pair of rows contains the eight patterns used in one of the four classification tasks.

C-response could be achieved by classifying the eight response cards into two groups of four cards differing in their apex or width of the angle. When only one stimulus card was present, a C-response was recorded when $\mathrm{S}$ sorted four appropriate cards in the black box (either in terms of the apex or the width of the angle) and the remaining four cards in the gray box.

An OD-response was an incomplete C-reponse which included the response card identical to the stimulus card, e.g., 1, 2, or 3 of the pointed angles including the one identical to the stimulus card displayed on the black box. An OG-response was an enlarged C-response which included all four response cards appropriate to the stimulus card plus some or all of the remaining response cards. An UC-response was one which did not meet the standards of a C-, OD-, or OG-response.

A modal response for each $\mathrm{S}$ was determined and from these data Fig. 2, which indicates the proportion of 


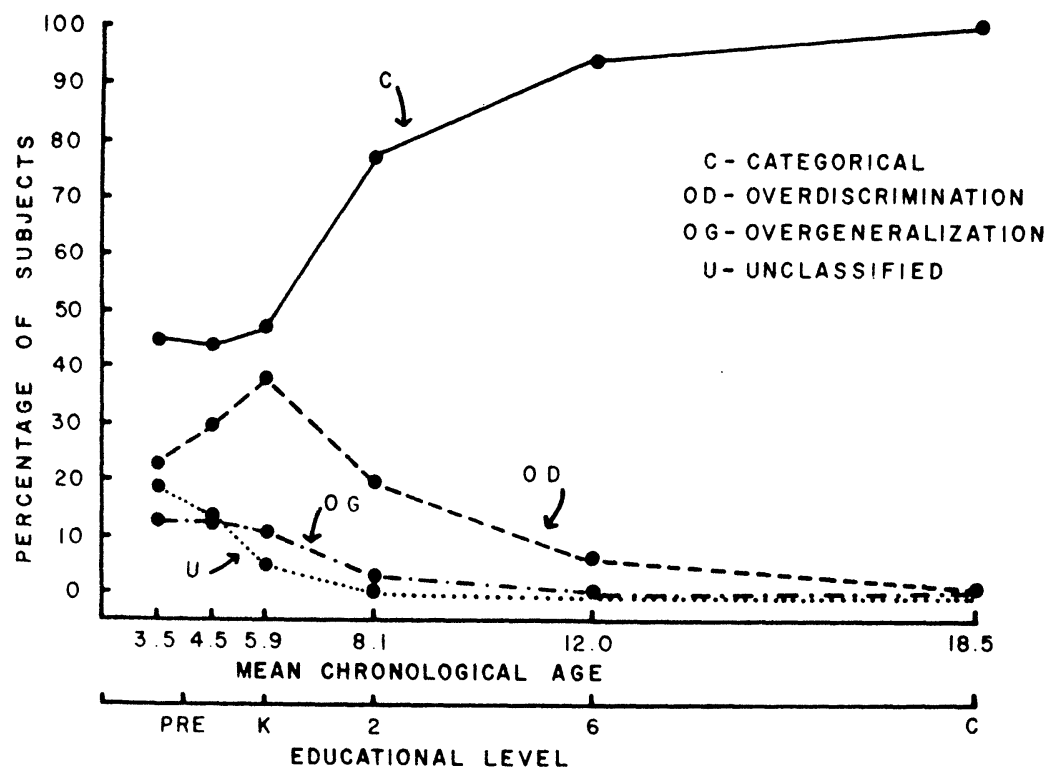

Fig. 2. A developmental analysis of free-classification behavior in terms of percentage of modal responses for different age groups.

each age group using the four different classification systems, was constructed. When a $\mathrm{S}$ made two classification responses equally often (e.g., two C- and two OD-responses) each response was assigned a value of $.5 \mathrm{~S}$ when computing the proportion of Ss using that response at that age level.

The change in frequency of each of these response measures with age was significant $\left[\chi^{2}(15)=58.50\right.$, $p<.0011$. Inspection of Fig. 2 suggests that $C$-responses increase with age, UC- and OG-responses decrease, while OD-responses first increase and then decrease. A chi-square analysis of OD-responses over age in comparison to all other responses was significant; $\left[\chi^{2}(5)\right.$ $=20.36, \mathrm{p}<.005]$. The linear and quadratic trends were both significant $\left[\chi^{2}(1)=12.19, \mathrm{p}<.001\right.$ and $\chi^{2}(1)=$ $5.45, \mathrm{p}<.025]$. (One must interpret these trend tests cautiously because of the violation of the assumption of equal intervals between each successive pair of age groups. Such an assumption is approximately correct for the log of the mean age in the three youngest groups, but the intervals between log of the mean age increases with the older groups.)

None of the variables (presence vs absence of stimulus cards, two vs three sorting categories, variation of stimulus cards in terms of one or two characteristics) other than age exerted a robust effect upon the classification behavior. For example, the evidence suggested that OD-responses were more common with three than with two sorting categories but nonmonotonic functions were obtained under both conditions.

\section{DISCUSSION}

The obtained results are consistent with the notion that classificatory behavior reflects a developmental sequence involving two successive behavioral mechanisms (Kendler, 1971). Initially a simple stimulus response (input-output) model, governs behavior via the mechanism of stimulus generalization, so that the S's classifications are directly dependent upon the characteristics of the stimulus. As a result, OG-responses are encouraged, and probably UC-responses as well, depending upon what feature of the stimulus attracts S's attention. Later a mediational mechanism (input-transformation-output model) operates so that the S's symbolic representational response to the stimuli controls behavior. During the early portion of this mediational stage, the S's conceptualizations are relatively narrow, thus encouraging OD-responses. Later the $S$ becomes capable of representing the entire category thus encouraging C-classifications.

Because the youngest $S s$ in the present experiment are beginning to be capable of operating mediationally, the obtained results would be expected: ontogenetic decreases in OG- and UC-responses, increases in C-responses, and an initial increase followed by a decrease in OD-responses. These results, however, must be considered only as suggestive. Further evidence is required before the obtained ontogenetic relationships can be accepted with confidence. In addition, the proposed model must be enlarged to include effects on classification behavior of stimulus material different from that used in the present experiment. Dimensional stimuli with psychophysical values would be particularly useful in increasing the precision of the present model.

\section{REFERENCES}

Dunn, L. D. Peabody picture vocabulary test (PPVT). Nashville, Tenn: American Guidance Service, 1959.

Kendler, H. H. Environmental and cognitive control of behavior. American Psychologist, 1971, 26, 962-973.

Kendler, H. H., \& Kendler, T. S. Vertical and horizontal processes in problem solving. Psychological R eview, 1962, 69, $1-16$.

Kendler, H. H., \& Kendler, T. S. Developmental processes in discrimination learning. Human Development, 1970, 13, 65-89.

Saltz, E., \& Siegel, I. E. Concept overdiscrimination in children. Journal of Experimental Psychology, 1967, 73, 1-8.

(R eceived for publication August 3, 1974.) 